\title{
Étude de l'activité antiamibienne des quelques plantes médicinales utilisées par les tradipraticiens de la ville de Lubumbashi, R.D.Congo.
}

\author{
Manya Tsheko ${ }^{1}$, Ndibualonji Badibanga Bualufu ${ }^{2}$, Maryabo Kazembe ${ }^{2}$ \\ Institut Supérieur de Techniques Médicales; B.P.4748, Lubumbashi, R D Congo \\ Université de Lubumbashi, Faculté de médecine Vétérinaire, B.P. 1825, Lubumbashi, R.D.Congo \\ Correspondance: Prof. Dr Victor Ndibualonji B.B. Téléphone: +243 814532349/ +243976734124, Email: \\ vndibualonji@hotmail.com
}

Original submitted in on $21^{\text {st }}$ February 2019. Published online at www.m.elewa.org/journals/ on $31^{\text {st }}$ May 2019 https://dx.doi.org/10.4314/jab.v137i1.3

\begin{abstract}
RESUME
Objectifs: Cette étude a été menée dans le but de rechercher les solutions phyto thérapeutiques aux problèmes posés par les diarrhées amibiennes en évaluant l'activité anti amibienne des plantes médicinales utilisées par les tradipraticiens de la ville de Lubumbashi.

Méthodologie et Résultats: Le protocole analytique comprend: les connaissances ethnobotaniques, le criblage chimique, les investigations mathématiques, la macération et les interactions prouvant l'adéquation ou non d'un éventuel traitement. Les cinq plantes les plus utilisées dans le traitement de diarrhée amibienne sont: Acalypha paniculata (queue de chat), Euphorbia hirta (herbe Australienne à asthme), Uapaca pilosa (palutivier), Uapaca bengoelensis (Alebié) et Psidium goyava (Goyavier). Les facteurs favorisant l'usage de ces plantes sont: l'accessibilité, la précarité des conditions de vie et l'urgence de traitement. Par le calcul de khi carré, il a été conclu qu'il n'existe pas de différences significatives d'effets thérapeutiques des extraits avec lesquels le traitement a eu lieu. L'antiamibiogramme a démontré la sensibilité d'Entamoeba histolytica vis-à-vis des extraits de quatre plantes à la concentration d'au moins $0,625 \mathrm{mg} / \mathrm{ml}$ sauf ceux de Psidium goyava

Conclusion et applications: L'activité antiamibienne a été prouvée pour toutes les espèces étudiées, sauf pour Psidium goyava. II a été mis en évidence la sensibilité de Entamoeba histolytica vis-à-vis des extraits de Acalypha paniculata, Euphorbia hirta, Uapaca bengoelensis et Uapaca pilosa. La plus Faible Concentration Inhibitrice des extraits observée en vingt-quatre heures est de 0,625 mg / ml pour Euphorbia hirta (Plante entière) et pour les feuilles de Acalypha paniculata. C 'est pourquoi nous encourageons l'utilisation par la population de Acalypha paniculata, Euphorbia hirta, Uapaca bengoelensis et Uapaca pilosa dans le traitement des diarrhées amibiennes
\end{abstract}

Mots clefs: Diarrhée - Entamoeba histolytica - antiamibiogramme 


\section{ABSTRACT}

Study of antiamibian activity of some medicinal plants used by traditional practitioners of the city of Lubumbashi, D R.Congo.

Objectives: This study was conducted in order to search for herbal solutions to the problems posed by amoebic diarrhea by evaluating the antimicrobial activity of medicinal plants used by traditional healers in Lubumbashi city.

Methodology and Results: The analytical protocol includes ethnobotanical knowledge, chemical screening, math investigations, maceration and interactions proving the adequacy or not of any treatment.

The five most commonly used plants in the treatment of amoebic diarrhea plants are: Acalypha paniculata, Euphorbia hirta, Uapaca bengoelensis, Uapaca pilosa and Psidium goyava. Factors encouraging the use of these plants are: accessibility, precarious living conditions and urgency of treatment. By calculating Chi square, it was concluded that there are no significant differences of therapeutic effects of the extracts with what the treatment took place.Susceptibility demonstrated a sensitivity of Entamoeba histolytica againt the extrats of four plants to the concentration of $0,625 \mathrm{mg} / \mathrm{ml}$ but not these of Psidium goyava.

Conclusion and Application of the Results: Antiamoebic activity was proven for all species studied except for Psidium goyava. The sensitivity of Entamoeba histolytica to extracts of Acalypha paniculata, Euphorbia hirta, Uapaca bengoelensis and Uapaca pilosa has been demonstrated. The lowest Inhibitory Concentration of the extracts observed in twenty-four hours is $0.625 \mathrm{mg} / \mathrm{ml}$ for Euphorbia hirta (whole plant) and for leaves of Acalypha paniculata. That is why we encourage by the population the use of Acalypha paniculata, Euphorbia hirta, Uapaca bengoelensis and Uapaca pilosa in the treatment of amoebic diarrhea.

Keywords: Diarrhea, Entamoeba histolytica, antiamibial test

\section{INTRODUCTION}

Les diarrhées sont parmi les manifestations pathologiques qui sévissent fréquemment dans les milieux urbains et ruraux en République Démocratique du Congo (D.R.Congo). A Lubumbashi, les études menées par le Système Nationale d'Informations Sanitaires (SNIS) ont enregistré de 2008 à 2009 les données suivantes : 9604 cas de diarrhée simple, 785 cas de diarrhée sanglante et 1423 cas de diarrhée amibienne. Le taux de prévalence de ces infection représente $9,17 \%$ de l'ensemble de pathologies les plus observées (paludisme 100507 cas soit $78 \%$; infection respiratoires aigües 10591 cas, soit 8\% ; IST 5826 cas, soit 4\%). Cependant, la ville de Lubumbashi compte au moins 329 établissements de soins (Lubaba, 2004). Ce réseau sanitaire devrait répondre aux besoins de patients en cas de pathologies à potentiel épidémique telles que les diarrhées. (Kakoma et al., 2004) constatent en outre que plus de $60 \%$ d'habitants de la ville de Lubumbashi consultent les tradipraticiens, et utilisent les extraits de plantes médicinales fournis par ces derniers pour le traitement des pathologies diarrhéiques. Devant cet état de la question, nous nous sommes préoccupés d'évaluer l'efficacité des extraits avec lesquels le traitement a eu lieu et de déterminer la sensibilité éventuelle de Entamoeba histolytica, agent causal des diarrhées amibiennes de manière à trouver des solutions pour endiguer ce type de pathologies (Lumbu et al., 2005). De toutes les études précitées, aucune n'a abordé les diarrhées amibiennes à part celle menée à Kinshasa par Mulumba (1999). La présente étude constitue une contribution au renforcement de capacité de lutte contre les pathologies à potentiel épidémique telles que les diarrhées amibiennes. 


\section{MATERIELS ET METHODES}

Milieu d'étude : Les enquêtes ethnobotaniques ont été réalisées dans la Ville de Lubumbashi, chef-lieu de la province du Katanga en RD Congo. Cette ville s'étend entre $11^{\circ} 37^{\prime}$ et $11^{\circ} 45^{\prime}$ de latitude Sud, $27^{\circ} 27^{\prime}$ et $27^{\circ} 32^{\prime}$ de longitude Est. Elle comprend sept communes administratives : Katuba, Kamalondo, Kampemba, Kenya, Lubumbashi, Rwashi et commune Annexe. La ville est située à une altitude moyenne de $1230 \mathrm{~m}$ caractérisée par un climat tropical humide à deux saisons: la saison pluvieuse s'étend de novembre à mars et la saison sèche de mai à septembre. Avril et octobre sont des mois de transition. La température moyenne annuelle est de l'ordre de $20^{\circ} \mathrm{C}$. Les températures les plus basses sont observées au début de la saison sèche de mi-mai à fin juillet. La savane arbustive prédomine, formant une brousse parsemée d'arbustes tels que les genres Uapaca, Psidium et Harungana (Manya, 2008).

Matériel: Pour cette expérimentation, nous avons utilisé le matériel suivant: la balance analytique APX200 Phywe, le balon à fond plat, l'étuve, l'évaporateur rotatif, les lames et les lamelles, le microscope binocculaire, le mortier, le pH mètre E520 Methron, le pilon, les pipettes pasteur, les tablettes de Ringer, les tubes à essai et les tubes à hémolyse. Pour la culture de Entamoeba histolytica, nous avons utilisé le milieu polyxènique de Dobel : l'amidon de riz, le blanc d'œuf, l'eau physiologique, le sang de cheval et la solution de Ringer.

Méthodologie du travail : La prospection a consisté à récolter les données ethnobotaniques auprès de 40 tradipraticiens de la ville de Lubumbashi et ses environs. Ces plantes ont été identifiées auprès des botanistes de la Faculté des Sciences de l'Université de Lubumbashi. Les fréquences de cessation des diarrhées « $D A$ » observées après traitement et celles dont les résultats du traitement sont inconnus « $R I$ » ont permis de recourir à l'expression mathématique de $X^{2}$ en prenant en considération le seuil de sécurité $95 \%$ et $99 \%$. Le décorticage, le séchage à l'ombre, le broyage, la pesée de la poudre végétale et l'évaporation douce

\section{RESULTATS}

L'identification des espèces végétales s'est faite sur base des données botaniques et des noms vernaculaires attribués à chacune des plantes. Acalypha paniculata nommé Kalungula en langue kaonde, Kabobo en langue bemba et Mutanda en langue lunda appartient à la famille de Euphorbiaceae. Euphorbia hirta appelé Magiba ya kalulu en langue à $50^{\circ} \mathrm{C}$ sous vide se sont déroulés au Laboratoire de la même faculté. La pesée de la poudre végétale a été réalisée à l'aide de la balance analytique APX-200 Phywe. $\mathrm{Le} \mathrm{pH}$ des extraits a été déterminé à l'aide $\mathrm{du}$ $\mathrm{pH}$ mètre E520 Métrons. La détermination de substances indicatrices de principes actifs s'est faite par des réactions de précipitation et de coloration (Sofowora, 1989; Lumbu et al., 2005). Pour l'expérimentation, une extraction a été réalisée avec l'eau (extrait brut), solvant avec lequel nous avons pratiqué la macération suivie de l'évaporation douce à $50^{\circ} \mathrm{C}$. Les dilutions successives d'ordre deux en partant de $2,5 \mathrm{mg} / \mathrm{ml}$ jusqu'à $0,625 \mathrm{mg} / \mathrm{ml}$ ont été opérées (données reprises dans le Tableau 3). Trois dilutions ont été retenues pour évaluer la sensibilité des germes vis-à-vis des extraits: $1 / 4 ; 1 / 2$ et 1 , donnant respectivement des concentrations de $0,625 \mathrm{mg} / \mathrm{ml}$; $1,25 \mathrm{mg} / \mathrm{ml}$ et $2,5 \mathrm{mg} / \mathrm{ml}$. L'agent causal de l'amibiase, Entamoeba histolytica, a été entretenu selon la technique polyxènique de Dobel (Riviere et al., 1987). C'est avec cette souche que l'antiamibiogramme a été réalisée au Laboratoire de Microbiologie de Cliniques Universitaires de Lubumbashi selon la technique décrite par Bailenger (1973) et Diamond et al. (1978). L'amibe dysentérique, Entamoeba histolytica, a été cultivé dans quatre tubes selon la technique diphasique de Dobel (Wery, 1995) . A l'aide d'une pipette pasteur, 1 goutte d'une solution de Ringer riche en amibes a été introduite dans des tubes contenant $4 \mathrm{ml} \mathrm{du}$ sang de chevaux coagulé (milieu polyxènique de Dobel). On introduit au premier tube $1 \mathrm{ml}$ d'extrait préalablement préparé. L'expérimentation s'est poursuivie en introduisant dans chacun de tubes $1 \mathrm{ml}$ d'extrait de différentes concentrations. Dans cette batterie, la concentration décroît de $2,5 \mathrm{mg} / \mathrm{ml}$ pour le $1^{\text {e }}$ tube jusqu'à $0,625 \mathrm{mg} / \mathrm{ml}$ pour le dernier tube d'épreuve. Tous les tubes ont été portés à l'étuve à $37^{\circ} \mathrm{C}$ pendant 24 heures. Le tube dans lequel on avait introduit aucun extrait a servi de témoin positif. L'estimation de l'activité inhibitrice s'est faite heure par heure au microscope à l'objectif $10 x$, puis 40x (Kambu et al., 1990).

swahili, Butovitonvi en langue tshiluba et Kake en langues tshokwe et bemba appartient aussi à la famille de Euphorbiaceae. Uapaca bengoelensis appelé Masuku en langue swahili, Mukuale en langue rund et Mupangwa ou Tuamba en langue bemba appartient aussi à la famille de Euphorbiaceae. Uapaca pilosa Hutch, appelé Mupangwa ou Masuku en langue bemba 
appartient aussi à la famille de Euphorbiaceae. Seule Psidium goyava appelé Mapela en langue lingala et swahili appartient à la famille de Myrtaceae. La numération suivante des plantes tient compte de leur disponibilité: (1) E. hirta, (2) P. goyava; (3) U. pilosa ; (4) $U$.bengoelensis et (5) A. paniculata.

Tableau 1 : Fréquences observées $(\mathrm{F})$ et Fréquences théoriques $(\S)$

\begin{tabular}{|l|l|l|l|l|l|l|l|l|}
\hline \multirow{4}{*}{$(\mathrm{F})$} & PLANTE : & $\mathbf{1}$ & $\mathbf{2}$ & $\mathbf{3}$ & $\mathbf{4}$ & $\mathbf{5}$ & TOTAL \\
\cline { 2 - 9 } & & & & & & & & \\
\cline { 2 - 9 } & & DA & 30 & 28 & 22 & 20 & 31 & 131 \\
\cline { 2 - 9 } & & RI & 102 & 57 & 62 & 61 & 89 & 371 \\
\hline \multirow{3}{*}{$(\S)$} & Total & 132 & 85 & 84 & 81 & 120 & 502 \\
\cline { 2 - 9 } & & DA & 34 & 22 & 22 & 21 & 31 & 130 \\
\cline { 2 - 9 } & & RI & 98 & 63 & 62 & 60 & 89 & 372 \\
\cline { 2 - 8 } & & Total & 132 & 85 & 84 & 81 & 120 & 502 \\
\hline
\end{tabular}

L'étude des fréquences classe les résultats du traitement de 502 patients en : patients dont la diarrhée s'est arrêtée après traitement « $\mathrm{DA}$ » et ceux dont les résultats du traitement sont inconnus « $\mathrm{Rl}$ ».

Tableau 2: Résultats du criblage chimique

\begin{tabular}{|c|c|c|c|c|c|c|c|c|c|c|c|c|c|}
\hline $\mathrm{N}^{\circ}$ & PLANTE & PU & \multicolumn{9}{|c|}{ Substances indicatrices des principes actifs } & $+/ p$ & Hen \\
\hline & & & $A l$ & Fla & Leu & Hca & Qun & Sap & Ste & Tan & Ter & & \\
\hline \multirow{3}{*}{1} & \multirow{3}{*}{ A.paniculata } & $\mathrm{F}$ & - & + & - & - & - & + & + & + & - & & - \\
\hline & & ET & - & - & - & - & - & - & + & + & - & 5 & - \\
\hline & & ER & - & - & - & - & + & + & - & + & - & & - \\
\hline \multirow[t]{2}{*}{2} & \multirow[t]{2}{*}{ E.hirta } & $\mathrm{PE}$ & - & - & - & - & - & + & - & + & - & 2 & - \\
\hline & & & & & & & & & & & & & \\
\hline \multirow{3}{*}{3} & \multirow{3}{*}{ U.bengoelensis } & $F$ & - & + & - & - & - & + & - & + & - & & - \\
\hline & & ET & - & - & + & + & - & + & + & + & +- & 8 & - \\
\hline & & ER & - & - & + & - & + & + & - & + & + & & - \\
\hline & & & & & & & & & & & & & \\
\hline \multirow{3}{*}{4} & \multirow{3}{*}{ U. pilosa } & $\mathrm{F}$ & - & + & - & - & - & + & - & + & - & & - \\
\hline & & ET & - & + & + & + & & + & + & + & - & 7 & - \\
\hline & & ER & - & + & + & & + & + & - & + & - & & - \\
\hline & & & & & & & & & & & & & \\
\hline \multirow{3}{*}{5} & \multirow{3}{*}{ P.goyava } & $F$ & - & + & + & - & - & - & - & + & - & 3 & - \\
\hline & & ET & - & - & - & - & - & - & - & - & - & & - \\
\hline & & $\mathrm{R}$ & - & - & - & - & - & - & - & - & - & & - \\
\hline & TOTAL & & 0 & 6 & 5 & 2 & 3 & 9 & 4 & 11 & 2 & $1 / I I$ & 0 \\
\hline
\end{tabular}

Légende: $P U=$ partie de la plante utilisée; Al=alcaloïdes; Fla=flavonoïdes; Leu=leucoantocyanes; Hca = hétérosides cardiotoniques; Qun =quinones; Sap=saponines; Ste=stéroïdes; Tan =tanins ; Ter=terpénoïdes; $H c n=h e ́ t e ́ r o s i d e s$ cyanogènes ; $\quad+/ P=$ résultats positifs par plante.

Uapaca bengoelensis, Uapaca pilosa et Acalypha indicatrices des principes actifs identifiées dans les paniculata possèdent au moins 5 substances parties utilisées. 
Tableau 3 : Interaction avec Entamoeba histolytica

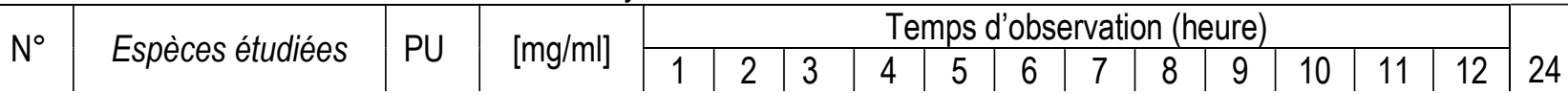

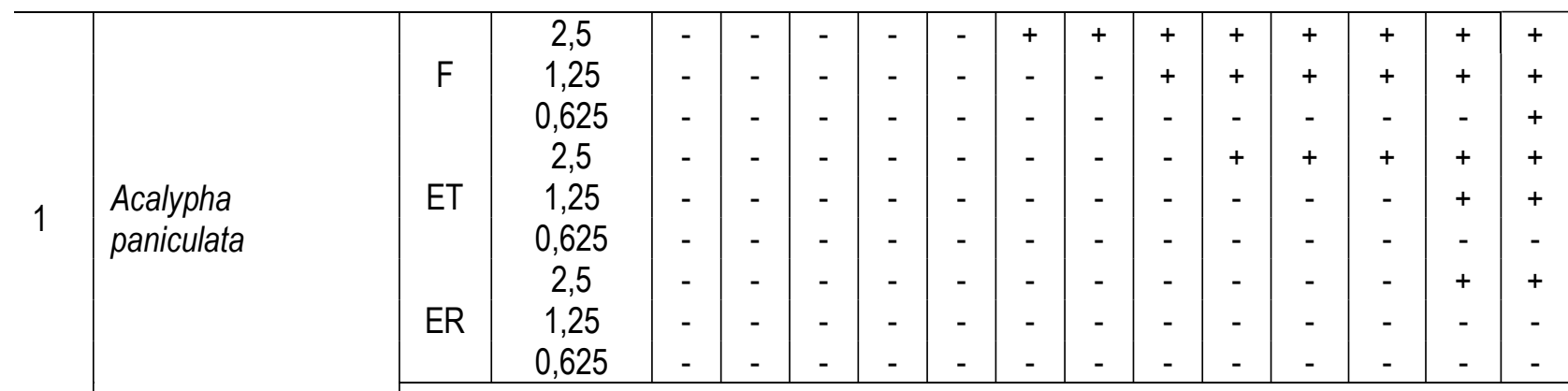

2

Euphorbia

hirta

Psidium

goyava

$\mathrm{PE} \mid$\begin{tabular}{c|c|c|c|c|c|c|c|c|c|c|c|c|c|c}
2,5 & - & - & + & + & + & + & + & + & + & + & + & + & + \\
1,25 & - & - & - & - & - & + & + & + & + & + & + & + & + \\
0,625 & - & - & - & - & - & - & - & - & - & - & - & + & + \\
\hline
\end{tabular}

Uapaca
bengoelensis

Uapaca
bengoelensis

\begin{tabular}{c|c|c|c|} 
& 2,5 & - & - \\
ET & 1,25 & - & - \\
0,625 & - & - \\
& 2,5 & - & - \\
2,5 & - & - \\
& 0,625 & - & - \\
& 2,5 & - & - \\
& 1,25 & - & - \\
0,625 & - & - & - \\
& 0,625 &
\end{tabular}

4

\begin{tabular}{|c|c|c|c|c|c|}
\hline \multirow{3}{*}{$F$} & 2,5 & - & - & - & - \\
\hline & 1,25 & - & - & - & - \\
\hline & 0,625 & - & - & - & - \\
\hline \multirow{3}{*}{ ET } & 2,5 & - & - & - & - \\
\hline & 1,25 & - & - & - & - \\
\hline & 0,625 & - & - & - & - \\
\hline \multirow{3}{*}{ ER } & 2,5 & - & - & - & - \\
\hline & 1,25 & - & - & - & - \\
\hline & 0,625 & - & - & - & - \\
\hline
\end{tabular}

$5 \quad$\begin{tabular}{|} 
Uapaca \\
Pilosa H
\end{tabular}

\begin{tabular}{|l|c|c|c|c|c|c|c|c|c|c|c|c|c|c|c}
\multirow{5}{*}{$F$} & 2,5 & - & - & - & - & - & - & - & - & - & - & - & + & + \\
& 1,25 & - & - & - & - & - & - & - & - & - & - & - & - & - \\
0,625 & - & - & - & - & - & - & - & - & - & - & - & - & - \\
& 2,5 & - & - & - & - & - & + & + & + & + & + & + & + & + \\
ET & 1,25 & - & - & - & - & - & - & - & - & - & - & - & - & + \\
0,625 & - & - & - & - & - & - & - & - & - & - & - & - & - \\
& 2,5 & - & - & - & - & - & - & - & + & + & + & + & + & + \\
\multirow{5}{*}{ ER } & 1,25 & - & - & - & - & - & - & - & - & - & - & - & + & + \\
& 0,625 & - & - & - & - & - & - & - & - & - & - & - & - & -
\end{tabular}

Légende $: \mathrm{PU}=$ partie utulisée $; \quad \mathrm{F}=$ feuille $; \quad \mathrm{ET}=$ écorse de tige $; \quad E R=$ écorse de racines et $\mathrm{PE}=$ plante entière. 
Les feuilles, les écorces de tige, les écorces de racines et les plantes entières ( $E$. hirta) constituent les parties utilisées pour le traitement de diarrhées amibiennes. La décoction est la forme galénique utilisée ; la boisson et

\section{DISCUSSION}

L'identification de ces espèces végétales en plusieurs langues montre que ces plantes sont suffisamment connues par plusieurs ethnies et probablement fréquemment utilisées (Kakez, 1999; Kahumba, 2000; Kabula, 2002).

Expression mathématique $X^{2}$ aux seuils de sécurité $95 \%$ et $99 \%$ :

Test de Khi-carré

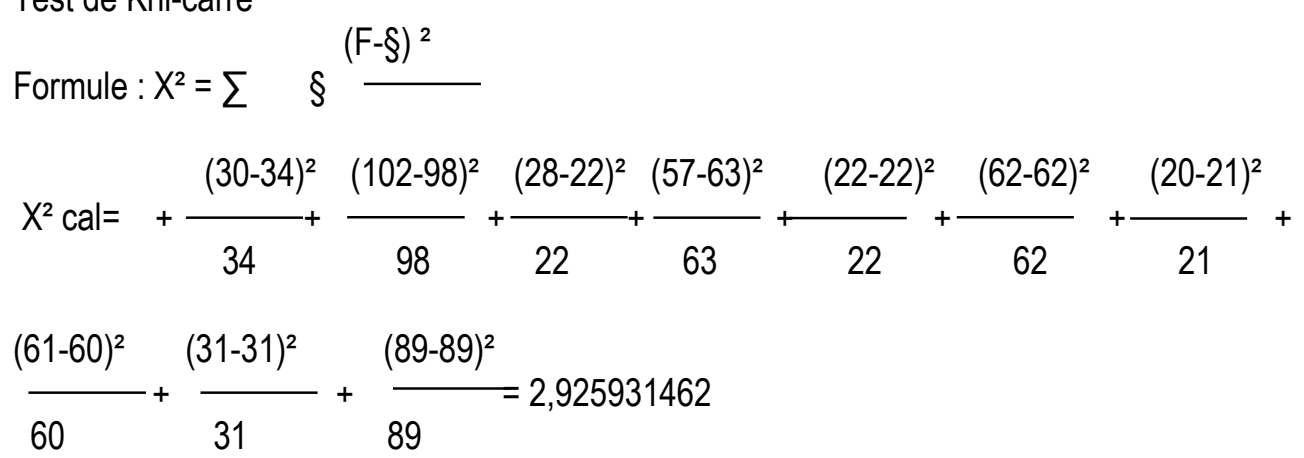

$\mathrm{X}^{2} \mathrm{cal}=2,92$

$X^{2}$ théorique : lecture dans le tableau de $X^{2}$

Degré de liberté : $U=(L-1)(C-1) \quad$ avec $L=$ ligne et $C=$ colonne $\mathrm{U}=(2-1)(5-1)=4$

Lecture : $X^{2}(0,05)=9,09$ et $\quad X^{2}(0,01)=11,28$

Conclusion : $2,92<9,09<11,28$

$X^{2}$ calculé est nettement inférieur à $X^{2}$ théorique.

Décision : l'hypothèse Ho est acceptable.

Donc, il n'existe pas de différence significative d'effets thérapeutiques. Toutes les quatre espèces possèdent d'effets thérapeutiques comparables à ceux de Euphorbia hirta. Au regard du Tableau 3, l'activité inhibitrice est observée à la concentration de $2,5 \mathrm{mg} / \mathrm{ml}$ à partir de 3 heures pour Euphorbia hirta, de 6 heures pour Acalypha paniculata et Uapaca pilosa et de 8 heures pour Uapaca bengoelensis. L'inhibition est aussi observée à la concentration de $1,25 \mathrm{mg} / \mathrm{ml}$ après 6 heures pour $E$. hirta et 8 heures pour $A$. paniculata. A $0,625 \mathrm{mg} / \mathrm{ml}$, l'action inhibitrice est observée une seule fois à partir de 12 heures et deux fois après 24 heures d'observation. L'espèce Psidium goyava n'a manifesté aucune activité vis-à-vis de Entamoeba histolytica. le purge sont les modes d'administration. L'interaction entre les extraits des plantes et Entamoeba histolytica s'est faite en fonction des concentrations des extraits.

Euphorbia hirta, l'une de cinq plantes, possède des vertus thérapeutiques antiamibiennes éprouvées (Abayonie, 1996; Kahumba, 2000).

Hypothèse nulle : Ho

" II n'existe pas de différence significative d'effets thérapeutiques quant aux extraits avec lesquels le traitement a eu lieu ». 
substances antiseptiques et diurétiques; des quinones, substances fongicides, antibiotiques, vermifuges, purgatives; des saponines qui sont des substances diurétiques, anti inflammatoires; des stéroïdes qui sont des régulateurs biologiques et des tanins qui sont anti diarrhéiques, anti septiques et anti venimeux. Ainsi nos résultats confirment les observations antérieures (Solomon, 1992; Charpentier, 1998; Anonyme, 2000). $A$. paniculata possède les flavonoïdes, les quinones, les saponines, les tanins et les stéroïdes tandis que $E$.

\section{CONCLUSION ET APPLICATION DES RESULTATS}

L'analyse du problème de santé relatif à l' amibiase, infection à potentiel épidémique, a permis d'envisager la présente étude en menant des expérimentations sur les espèces végétales présumées antiamibiennes. Par le calcul de Khi carré, il a été démontré qu'il n'existait pas de différence significative quant à l'efficacité des extraits avec lesquels les traitements ont eu lieu. Pour avoir un apriori sur l'efficacité des effets thérapeutiques des extraits utilisés, un screening chimique de différents organes des plantes a été effectué. L'activité antiamibienne a été prouvée pour toutes les espèces étudiées sauf pour Psidium goyava. II a été mis en

\section{BIBLIOGRAPHIE}

Abayomi S.: Plantes médicinales et médecine traditionnelle d'Afrique, Ed. Karthala, Paris, 1996, pp 290-297

Anonyme : Répertoire des médicaments, Centre belge d'informations pharmaceutiques, 14ème Ed., Bruxelles, 2000, pp 216-217

Bailenger J.: Coprologie parasitaire et fonctionnelle, Ed. Drouillard, Bordeaux, 1973 pp 175-179

Bruneton J : Pharmacognosie, Ed Tec \& Doc, Paris, 2009, pp 22, 365-366, 441.

Charpentier B.: Guide pratique du préparateur en pharmacie, Ed. Masson, Paris, 1998, pp 10531059, 1062

Diamond L. S.; Harlow DR.; Cunnineck CC.: A new medium for the axenic cultivation of Entamoeba, Transaction of the royal society of tropical medicine and hygiene, 1978, 72: 432 439.

Kabyla I. Introduction à la santé publique; Presses Universitaires de Lubumbashi, 2002, pp.11-32.

Khalid S.A. et Dullick H.: Isolation and characterisation of an antimalarial agent of the neem tree Azadiratcha indica; Journal of natural products, 1989, 52 (5): 922-927. hirta contient des saponines, des tanins et des flavonoïdes. Le nombre des groupes de substances ne semblent pas tellement jouer car sur ce plan, les deux espèces de Uapaca en possèdent au moins sept sans pour autant manifester le plus d'activité antiamibienne. En effet, la qualité de substances et leurs concentrations seraient probablement les facteurs déterminants (Lambo, 1979; Khaid et Dullick, 1989; Bruneton, 2009).

évidence la sensibilité de Entamoeba histolytica vis-àvis des extraits de Acalypha paniculata, Euphorbia hirta, Uapaca bengoelensis et Uapaca pilosa. La plus Faible Concentration Inhibitrice des extraits observée en vingt-quatre heures est de $0,625 \mathrm{mg} / \mathrm{ml}$ pour Euphorbia hirta (Plante entière) et pour les feuilles de Acalypha paniculata. C'est pourquoi nous encourageons l'utilisation par la population congolaise en général et Lushoise en particulier de Acalypha paniculata, Euphorbia hirta, Uapaca bengoelensis et Uapaca pilosa dans le traitement des diarrhées amibiennes.

Kahumba B. : Contribution à l'étude des Euphorbiaceae utilisés contre les infections bactériennes à Lubumbashi, Mémoire de DEA en Sciences pharmaceutiques, UNILU, 2000, pp 48-51

Kakez K.: Notes d'Anthropologie médicale; PUL, Lubumbashi, 1999 ; pp.6, 12-13, 19-30.

Kakoma S. ; Lumbu S.; Peti P. ; Vyakanakazi M.D. ; Ngoy K.E. ; Kalonda M.E. ; Mbayo K.M. ; Kahumba B.; Shengo L.M. et Kayembe K. (2004): Bunganga ya mici: Rapport de recherche effectuée durant la deuxième session des travaux de l'observatoire de changement urbain, PUL, Lubumbashi, pp 115-125

Kambu K., Tona L., Kaba S., Cimanga K. et Mukala N. : Activité antispasmodique d'extraits à partir de plantes utilisées en préparations comme anti diarrhéiques à Kinshasa, Zaire. Ann. Pharmaceutiques françaises, Ed Masson, Paris, 1990,48(4): 200-208.

Lambo J.O.: The healing powers of herbs, with special reference to obstetrics and gynecology, African medicinal plants (Sofowra E.A), University of the Ife Presse, Nigeria, 1979, pp.23-31. 
La Rivière, M., Beauvais B., Derouin, F., Traoré, F. : Parasitologie médicale; Ed. Ellipse; Poitier, 1987 ; pp.67, 72-73.

Lubaba B.N.: Exposition au Plomb et au Cadmium dans la ville de Lubumbashi, thèse de doctorat en Santé Publique, Université de Lubumbashi, Lubumbashi, 2004.

Lumbu S; Penge O., Kahumba B., Kalonda M. et Mbayo K.: Contribution à l'étude des quelques plantes médicinales anti diarrhéiques en usage dans la ville de Lubumbashi et ses environs, Annales de pharmacie, Unikin, 2005, vol. 13(1) pp 75-86

Manya T. : Etude de l'activité antiamibienne des plantes médicinales utilisées à Lubumbashi, Mémoire de DEA, Université de Lubumbashi, Lubumbashi, 2008, pp 57-61

Mulumba M.: Eléments de Protozoologie médicale; Ed. Biometrix, Kinshasa, 1999, pp 32, 42-43

Sofowora E.A.: Pharmacognosies, Bailleur Tindal Itd, Londres, 1989.

Solomon G.: Organic chemistry, $15^{\text {th }}$ ed, New York, 1992, pp 950, 1053

Wery M.: Protozoologie médicale, Ed. De Boeck, Bruxelles, 1995, pp225-226 